\title{
SMALL INTO-ISOMORPHISMS BETWEEN SPACES OF CONTINUOUS FUNCTIONS
}

\author{
Y. BENYAMINI ${ }^{1}$
}

\begin{abstract}
We prove that if $K$ is a compact metric space, $0<\varepsilon<1$, and $T$ is an operator from $C(K)$ into $C(S)$ satisfying $\|f\|<\|T f\|<(1+\varepsilon)\|f\|$ for all $f \in$ $C(K)$, then there is an isometry $W$ of $C(K)$ into $C(S)$ with $\|T-W\|<3 e$. We also give an example to show that this is no longer true when $K$ is not assumed to be metrizable.
\end{abstract}

Introduction. In [1], [3] D. Amir and M. Cambern proved, independently, that if $C(K)$ and $C(S)$ are isomorphic with an isomorphism $T$ satisfying $\|T\|\left\|T^{-1}\right\|<2$, then $K$ and $S$ are homeomorphic. Hence $C(K)$ and $C(S)$ are in fact isometric. Their proofs (especially the proof in [3]) give in fact more: If $\|T\|\left\|T^{-1}\right\|=1+\varepsilon$ (with $0<\varepsilon<1$ ), then an isometry $W$ can be chosen close to $T$, with $\|T-W\|$ depending only on $\varepsilon$.

In this article we study the same problem for into-isomorphism: Does there exist a function $g(\varepsilon)$ defined for small enough $\varepsilon>0$, with $\lim _{\varepsilon \rightarrow 0} g(\varepsilon)=0$, such that if $T$ : $C(K) \rightarrow C(S)$ is an into-isomorphism satisfying $\|T\|\left\|T^{-1}\right\|<1+\varepsilon$, then there is an isometry $W$ of $C(K)$ into $C(S)$ satisfying $\|T-W\|<g(\varepsilon)$.

It turns out that the answer is positive when $K$ is metrizable. This is Theorem 2 in $\S 2$. When $K$ is nonmetrizable this is no longer true. A counterexample is given in $\S 3$.

The proof of Theorem 2 depends on the construction of a special $w^{*}$-continuous retract on the unit ball of $C(K)^{*}$. This subject is treated in $\S 1$. The last section contains some remarks and open problems.

We shall use standard notation, see e.g. [6]. The reader is also referred to [6] and its references for results quoted without a specific reference.

We shall treat the real case only. Some modifications are needed in the complex case, and the estimates obtained are somewhat worse than in the real case.

I wish to thank D. Amir and Y. Sternfeld for many discussions during the preparation of this article.

1. Construction of a retract. Let $K$ be a compact metric space. In this section we construct a $w^{*}$-continuous retract on the unit ball of $C(K)^{*}$, which behaves "nicely" with respect to the norm topology. (See also (1) in §4.)

Received by the editors November 13, 1980 and, in revised form, February 20, 1981.

1980 Mathematics Subject Classification. Primary 46E15.

${ }^{1}$ This research was partially supported by the Fund for the Promotion of Research at the Technion, Israel Institute of Technology. 
Proposition 1. Let $K$ be a compact metric space. There is a $w^{*}$-continuous retract $r$ from $\left\{\mu \in C(K)^{*}:\|\mu\| \leqslant 3\right\}$ onto the unit ball of $C(K)^{*}$ satisfying $\|r(\mu)-\mu\|<$ $\|\mu\|-1$ whenever $\|\mu\| \geqslant 1$.

Proof. We first note that it is enough to prove the proposition for $K=\Delta$-the Cantor set. Indeed, assume $s$ is a retract on the unit ball of $C(\Delta)^{*}$ satisfying the requirement of the proposition. By Milutin's Lemma, every $C(K)$, with $K$ compact metric, can be embedded as a subspace of $C(\Delta)$ with a norm one projection $P$ from $C(\Delta)$ onto $C(K)$. Given any $\mu \in C(K)^{*},\|\mu\| \leqslant 3$, we define $r(\mu)=\left.s\left(P^{*} \mu\right)\right|_{C(K)}$. It follows immediately from the $w^{*}$-continuity of $P^{*}$, and the fact that $\left\|P^{*}\right\|=\|P\|$ $=1$, that $r$ is the required retract.

We now turn to the proof of the proposition for $C(\Delta)$. Let $\left\{\Delta_{n, i}\right\}_{i=1, \ldots, 2^{n}}$ be the natural partition of $\Delta$ to $2^{n}$ disjoint closed and open sets with $\Delta_{n, i}=\Delta_{n+1,2 i-1} \cup$ $\Delta_{n+1,2 i}$. If $\mu \in C(\Delta)^{*}$, we put $\mu_{n, i}=\left.\mu\right|_{\Delta_{n, i}}$, i.e. $\mu_{n, i}(E)=\mu\left(E \cap \Delta_{n, i}\right)$ for all Borel subsets $E$ of $\Delta$.

Let

$$
A_{n, i}=\left\{\mu \in C(\Delta),\|\mu\| \leqslant 3: \sum_{j<i}\left[\left|\mu\left(\Delta_{n+1,2 j-1}\right)\right|+\left|\mu\left(\Delta_{n+1,2 j}\right)\right|\right]+\sum_{j>i}\left|\mu\left(\Delta_{n, j}\right)\right|<1\right\} .
$$

Since each $\Delta_{n, i}$ is closed and open, the sets $A_{n, i}$ are $w^{*}$-closed.

If $\mu \in A_{n, i} \backslash A_{n, i+1}$ (to save cumbersome notation, we denote here and in the following, $A_{n+1,1}$ by $A_{n, 2^{n}+1}$ when convenient) then necessarily $\mu\left(\Delta_{n+1,2 i-1}\right)$ and $\mu\left(\Delta_{n+1,2 i}\right)$ have different signs, and there is a unique $t$ so that

$$
\begin{aligned}
\left|\mu\left(\Delta_{n+1,2 i-1}\right)+t\right| & +\left|\mu\left(\Delta_{n+1,2 i}\right)-t\right| \\
& +\sum_{j<i}\left[\left|\mu\left(\Delta_{n+1,2 j-1}\right)\right|+\left|\mu\left(\Delta_{n+1,2 j}\right)\right|\right]+\sum_{j>i}\left|\mu\left(\Delta_{n, j}\right)\right|=1 .
\end{aligned}
$$

We define a retract $\varphi_{n, i}$ of $A_{n, i}$ onto $A_{n, i+1}$ by

$$
\varphi_{n, i}(\mu)=\mu \text { for } \mu \in A_{n, i+1} \text {, }
$$

and

$$
\varphi_{n, i}(\mu)=\frac{\mu\left(\Delta_{n+1,2 i-1}\right)+t}{\mu\left(\Delta_{n+1,2 i-1}\right)} \mu_{n+1,2 i-1}+\frac{\mu\left(\Delta_{n+1,2 i}\right)-t}{\mu\left(\Delta_{n+1,2 i}\right)} \mu_{n+1,2 i}+\sum_{j \neq i} \mu_{n, j}
$$

when $\mu \in A_{n, i} \backslash A_{n, i+1}$.

It is easy to check that $\varphi_{n, i}$ is $w^{*}$-continuous, and that

(a) $\varphi_{n, i}(\mu)\left(\Delta_{m, j}\right)=\mu\left(\Delta_{m, j}\right)$ for all $m \leqslant n$ and all $j=1, \ldots, 2^{m}$.

(b) $\left\|\varphi_{n, i}(\mu)-\mu\right\|=\|\mu\|-\left\|\varphi_{n, i}(\mu)\right\|$.

Also for $\mu \in A_{n, i} \backslash A_{n, i+1}$ we have

(c)

$$
\left\|\varphi_{n, i}(\mu)\right\|>\sum_{j<i}\left[\left|\varphi_{n, i}(\mu)\left(\Delta_{n+1,2 j-1}\right)\right|+\left|\varphi_{n, i}(\mu)\left(\Delta_{n+1,2 j}\right)\right|\right]+\sum_{j>i}\left|\varphi_{n, i}(\mu)\left(\Delta_{n, j}\right)\right|=1 \text {. }
$$

Similarly, we define a $w^{*}$-continuous retract $\varphi_{0}$ from $\left\{\mu \in C(\Delta)^{*}:\|\mu\|<3\right\}$ onto $A_{0,1}=\left\{\mu \in C(\Delta)^{*}:\|\mu\|<3, \mu(\Delta)<1\right\}$ by $\varphi_{0}(\mu)=\mu$ for $\mu \in A_{0,1}$, and $\varphi_{0}(\mu)=\mu /|\mu(\Delta)|$ when $\mu \notin A_{0,1}$. Again it is easy to see that $\varphi_{0}$ satisfies (b), and the analogue of (c), i.e. $\left\|\varphi_{0}(\mu)\right\|>\left|\varphi_{0}(\mu)(\Delta)\right|=1$ whenever $\mu \notin A_{0,1}$. 
For each $k=1,2, \ldots$, write $k=2^{n}+i$ with $1<i<2^{n}$, and let $\psi_{k}=$ $\varphi_{n, i} \circ \cdots \circ \varphi_{n, 1} \circ \varphi_{n-1,2^{n-1}} \circ \cdots \circ \varphi_{0,1} \circ \varphi_{0}$.

The functions $\psi_{k}$ are $w^{*}$-continuous, and we shall show that they converge to the desired retract $r$.

The metric $d(\mu, \nu)=\sum_{n=0}^{\infty} 4^{-n} \sum_{i-1}^{2^{n}}\left|(\mu-\nu)\left(\Delta_{n, i}\right)\right|$ induces the $w^{*}$-topology on $\left\{\mu \in C(\Delta)^{*}:\|\mu\| \leqslant 3\right\}$. By (a) $d\left(\psi_{k}(\mu), \psi_{l}(\mu)\right)<2^{-n}$ whenever $l>k>2^{n+2}$. Thus $\left\{\psi_{k}\right\}$ is a uniform Cauchy sequence, and it converges uniformly to a function $r$, which is thus $w^{*}$-continuous.

Since $\sum_{i=1}^{2^{n}}\left|r(\mu)\left(\Delta_{n, i}\right)\right|=\lim _{k} \sum_{i=1}^{2^{n}}\left|\psi_{k}(\mu)\left(\Delta_{n, i}\right)\right|<1$ for all $n$, we have that $\|r(\mu)\|$ $<1$ for all $\mu$, and obviously $r(\mu)=\mu$ whenever $\|\mu\|<1$. Thus $\mu$ is a retract.

It remains to estimate $\|\mu-r(\mu)\|$. Since $\psi_{k}(\mu) \stackrel{w^{*}}{\rightarrow} r(\mu)$, we have $\|r(\mu)-\mu\| \leqslant \lim \left\|\psi_{k}(\mu)-\mu\right\|$, but by (b), and the fact that each $\psi_{l}$ is obtained from $\psi_{l-1}$ by composition with some $\varphi_{n, i}$, we get (denoting $\mu$ by $\psi_{0}(\mu)$ )

$$
\begin{aligned}
\left\|\mu-\psi_{k}(\mu)\right\| & \leqslant \sum_{l=1}^{k}\left\|\psi_{l-1}(\mu)-\psi_{l}(\mu)\right\| \\
& =\sum_{l=1}^{k}\left[\left\|\psi_{l-1}(\mu)\right\|-\left\|\psi_{l}(\mu)\right\|\right]=\|\mu\|-\left\|\psi_{k}(\mu)\right\| .
\end{aligned}
$$

It follows from (c) that if $\|\mu\| \geqslant 1$, then $\left\|\psi_{k}(\mu)\right\|>1$ for all $k$, and thus $\| \mu-$ $\psi_{k}(\mu)\|<\| \mu \|-1$.

2. The metrizable case. In this section we prove the following

THEOREM 2. Let $K$ be a compact metric space, and let $0<\varepsilon<1$. Assume that $T$ : $C(K) \rightarrow C(S)$ satisfies $\|f\| \leqslant\|T f\| \leqslant(1+\varepsilon)\|f\|$ for all $f \in C(K)$. Then there is an isometry $W$ of $C(K)$ into $C(S)$ with $\|T-W\| \leqslant 3 \varepsilon$.

Since the range of $T$ is separable, it is contained in a subspace of $C(S)$, isometric to $C\left(S^{\prime}\right)$ with $S^{\prime}$ metrizable. Thus we shall assume below that $S$ is itself metrizable.

The next lemma is similar to the arguments in [3].

Lemma 3. Let $K, S, T$ and $\varepsilon$ be as above. For each $k \in K$, let $S(k)=\{s \in S$ : $\left.\left\|T^{*} \delta_{s}-\eta(s) \delta_{k}\right\| \leqslant \varepsilon\right\}$, where $\eta(s)=\operatorname{sign}(T 1)(s)$. Then

(i) $S(k) \neq \varnothing$ for all $k \in K$.

(ii) $S\left(k_{1}\right) \cap S\left(k_{2}\right)=\varnothing$ whenever $k_{1} \neq k_{2}$.

(iii) Each $S(k)$, and also $S_{1}=\cup S(k)$ are closed.

(iv) The mapping $\psi: S_{1} \rightarrow K$ defined by $\psi(s)=k$ iff $s \in S(k)$ is continuous.

Proof. Fix any $k \in K$. We start by showing that $\sup _{s}\left|T^{*} \delta_{s}\{k\}\right|>1$. Choose $f_{n} \in C(K)$ so that $\left\|f_{n}\right\|=1, f_{n}(k)=1$ and $f_{n}$ converge pointwise to the indicator function of $\{k\}$. Thus $T^{*} \delta_{s}\left(f_{n}\right) \rightarrow T^{*} \delta_{s}\{k\}$ for all $s \in S$.

Let $\mu$ be a Hahn-Banach extension of $T^{*-1} \delta_{k}$ from the image of $T$ to all of $C(S)$, i.e. $\mu \in C(S)^{*},\langle T f, \mu\rangle=f(k)$ for all $f \in C(K)$, and $\|\mu\|=\left\|T^{*-1} \delta_{k}\right\|<\left\|T^{-1}\right\|<$ 1. By the Dominated Convergence Theorem we have $1=f_{n}(k)=\left\langle T f_{n}(s), \mu(d s)\right\rangle$ $=\left\langle T^{*} \delta_{s}\left(f_{n}\right), \mu\right\rangle \rightarrow\left\langle T^{*} \delta_{s}\{k\}, \mu\right\rangle$. Since $\|\mu\|<1$ we indeed must have that $\sup _{s}\left|T^{*} \delta_{s}\{k\}\right| \geqslant 1$. 
Fixing any $s$ so that $\left|T^{*} \delta_{s}\{k\}\right|>(1+\varepsilon) / 2$, we shall show that $T^{*} \delta_{s}\{k\}$ has the same sign as $(T 1)(s)$. Indeed $(T 1)(s)=T^{*} \delta_{s}(K)=T^{*} \delta_{s}\{k\}+T^{*} \delta_{s}(K \backslash\{k\})$, but $\left\|T^{*} \delta_{s}\right\| \leqslant 1+\varepsilon$ implies that

$$
\left|T^{*} \delta_{s}(K \backslash\{k\})\right|=\left\|T^{*} \delta_{s}\right\|-\left|T^{*} \delta_{s}\{k\}\right|<(1+\varepsilon)-\frac{1+\varepsilon}{2}<\left|T^{*} \delta_{s}\{k\}\right| .
$$

Given now any $n>2 /(1-\varepsilon)$, if $s_{n}$ satisfies $\left|T^{*} \delta_{s_{n}}\{k\}\right|>1-1 / n$, then by the above, sign $T^{*} \delta_{s_{n}}\{k\}=\eta\left(s_{n}\right)$, hence $\left\|T^{*} \delta_{s_{n}}-\eta\left(s_{n}\right) \delta_{k}\right\|<\left\|T^{*} \delta_{s_{n}}\right\|-1+1 / n<$ $\varepsilon+1 / n$. Thus if $s$ is any limit point of the $\left\{s_{n}\right\}$,

$$
\left\|T^{*} \delta_{s}-\eta(s) \delta_{k}\right\| \leqslant \varlimsup \lim \left\|T^{*} \delta_{s_{n}}-\eta\left(s_{n}\right) \delta_{k}\right\|<\varepsilon,
$$

i.e. $s \in S(k)$.

This proves (i), and (ii) is obvious.

Assume now that $s_{n} \in S\left(k_{n}\right)$, and $s_{n} \rightarrow s$. By passing to a subsequence, assume that $k_{n} \rightarrow k$, hence $\left\|\eta(s) \delta_{k}-T^{*}\left(\delta_{s}\right)\right\|<\underline{\lim }\left\|\eta\left(s_{n}\right) \delta_{k_{n}}-T^{*} \delta_{s_{n}}\right\|<\varepsilon$, i.e. $s \in S(k)$. This proves both claims of (iii) as well as (iv).

Proof of Theorem 2. By Lemma 3, there is a closed subset $S_{1}$ of $S$, a continuous map $\psi$ from $S_{1}$ onto $K$, and a continuous \pm -valued function $\eta(s)$ so that $\left\|T^{*} \delta_{s}-\eta(s) \delta_{\psi(s)}\right\| \leqslant \varepsilon$ for all $s \in S_{1}$.

We shall find a $w^{*}$-continuous function $\xi: S \rightarrow C(K)^{*}$ satisfying

(1) $\|\xi(s)\| \leqslant 1$ for all $s \in S$.

(2) $\xi(s)=\eta(s) \delta_{\psi(s)}$ for all $s \in S_{1}$.

(3) $\left\|\xi(s)-T^{*} \delta_{s}\right\|<3 \varepsilon$ for all $s \in S$.

Once $\xi$ is constructed, we define $(W f)(s)=\langle f, \xi(s)\rangle$. For every $f \in C(K), W f \in$ $C(S)$ by the $w^{*}$-continuity of $\xi$, and $W$ is, of course, linear. By (1), (2) and the fact that $\psi$ is onto we see that $\|W f\|=\|f\|$ for all $f \in C(K)$, i.e. $W$ is an isometry. Finally, by (3) we have, for all $f \in C(K)$ and $s \in S$, that

$$
|(T-W) f(s)|=\left|\left\langle T^{*} \delta_{s}-\xi(s), f\right\rangle\right| \leqslant\left\|T^{*} \delta_{s}-\xi(s)\right\|\|f\| \leqslant 3 \varepsilon\|f\|,
$$

i.e. $\|T-W\| \leqslant 3 \varepsilon$.

Let $\phi$ be the set-valued function, defined for $s \in S$, and taking values in $C(K)^{*}$, given by

$$
\phi(s)= \begin{cases}\eta(s) \delta_{\psi(s)}, & s \in S_{1}, \\ \left\{\mu \in C(K)^{*}:\left\|\mu-T^{*} \delta_{s}\right\|<\varepsilon\right\}, & s \notin S_{1} .\end{cases}
$$

For each $s \in S, \phi(s)$ is a $w^{*}$-compact and convex subset of $C(K)^{*}$, and $\phi$ is lower-semicontinuous. Indeed, if $s_{n} \rightarrow s$ and $\nu \in \phi(s)$, define

$$
\nu_{n}= \begin{cases}\eta\left(s_{n}\right) \delta_{\psi\left(s_{n}\right.}, & s_{n} \in S_{1}, \\ T^{*} \delta_{s_{n}}-T^{*} \delta_{s}+\nu, & s_{n} \notin S_{1}\end{cases}
$$

Then $\nu_{n} \stackrel{w^{*}}{\rightarrow}$, and $\nu_{n} \in \phi\left(s_{n}\right)$. By Michael's selection theorem $\phi$ admits a $w^{*}$ continuous selection $\varphi$.

Let $r$ be the retract of Proposition 1, and define $\xi=r \circ \varphi$.

For each $s \in S,\|\varphi(s)\| \leqslant \varepsilon+\left\|T^{*} \delta_{s}\right\|<1+2 \varepsilon<3$. Thus $\xi$ is well defined, and clearly satisfies (1) and (2). To check (3), we have

$$
\left\|\xi(s)-T^{*} \delta_{s}\right\|=\left\|r(\varphi(s))-T^{*} \delta_{s}\right\|<\left\|T^{*} \delta_{s}-\varphi(s)\right\|+\|\varphi(s)-r(\varphi(s))\| .
$$


The first term is bounded by $\varepsilon$ by the choice of $\varphi$, while Proposition 1 gives that

$$
\|\varphi(s)-r(\varphi(s))\|<\|\varphi(s)\|-1 \leqslant 1+2 \varepsilon-1=2 \varepsilon \text {. }
$$

3. A counterexample. Theorem 2 is no longer true if we drop the assumption that $K$ is metrizable. In this section we describe a counterexample.

TheOREM 4. For every $\varepsilon>0$, there are compact Hausdorff spaces $K_{e}, S_{e}$, and an isomorphism $T: C\left(K_{\varepsilon}\right) \rightarrow C\left(S_{e}\right)$ satisfying $\|f\| \leqslant\|T f\|<(1+\varepsilon)\|f\|$, so that $\|T-W\|>2$ for all isometries $W$ of $C\left(K_{e}\right)$ into $C\left(S_{e}\right)$.

Proof. Let $A$ be the one point compactification of an uncountable discrete set $D$, and denote by $p$ the point of infinity. Fix $N$ so that $(N-1) \varepsilon>2$ and let $K=K_{\varepsilon}=A^{N}$. Identifying $k \in K$ with $\delta_{k} \in C(K)^{*}$, let $S=S_{e} \subset C(K)^{*}$ be defined by $S=K \cup\left\{\xi\left(a_{1}, \ldots, a_{N}\right): a_{i} \in A\right\}$ where

$$
\xi\left(a_{1}, \ldots, a_{N}\right)=\frac{1}{N-1} \sum_{i=1}^{N} \delta_{\left(a_{1}, \ldots, a_{i-1}, p, a_{i+1}, \ldots, a_{N}\right)}-\frac{1}{N-1} \delta_{(p, \ldots, p)} .
$$

$S$ is a $w^{*}$-closed subset of $C(K)^{*}$, and we define $T: C(K) \rightarrow C(S)$ by $T f(s)=$ $\langle f, s\rangle$. Since $S \supset K$, we have $\|T f\| \geqslant\|f\|$ for all $f \in C(K)$. Also $\|T f\|<$ $(1+\varepsilon)\|f\|$ because $S$ is contained in the ball of radius $(N+1) /(N-1)<1+\varepsilon$ around the origin. Note also that $T^{*} \delta_{s}=s$ for all $s \in S$.

We shall prove that there is no isometry $W: C(K) \rightarrow C(S)$ with $\|T-W\|<2$. Indeed, if $W$ is an isometry satisfying $\|T-W\|<2$, then the function $\varphi(s)=$ $W^{*} \delta_{s}$ is a $w^{*}$-continuous function satisfying

(1) $\{\varphi(s): s \in S\}$ is a one-norming subset of the unit ball of $C(K)^{*}$.

(2) $\|\varphi(s)-s\| \leqslant 2$ for all $s \in S$.

(To see (2), recall that $s=T^{*} \delta_{s}$, thus $\|\varphi(s)-s\|=\left\|\left(W^{*}-T^{*}\right) \delta_{s}\right\|<\|W-T\|$ $<2$.) We shall show that no $w^{*}$-continuous function $\varphi$, satisfying (1) and (2) exists.

Note first that for a $w^{*}$-closed subset of the unit ball of $C(K)^{*}$ to be onenorming, it must contain, for each $k \in K$, either the measure $\delta_{k}$ or $-\delta_{k}$. Thus $\{\varphi(s)$ : $s \in S\}$ must contain, for each $\bar{b}=\left(b_{1}, \ldots, b_{N}\right) \in D^{N}$ one of the points $\pm \delta_{\bar{b}}$, i.e. there is a point $s \in S$ with $\varphi(s)= \pm \delta_{\bar{b}}$. But by (2) $\|s-\varphi(s)\|<2$, and the only point $s \in S$ for which this is possible is $s=\delta_{\bar{b}}$. Thus $\varphi$ necessarily satisfies $\varphi\left(\delta_{\bar{b}}\right)=\delta_{\bar{b}}$ for all $\bar{b} \in D^{N}$. Since $D^{N}$ is dense in $K=A^{N}$, and $\varphi$ is continuous, we have, in fact $\varphi\left(\delta_{k}\right)=\delta_{k}$ for all $k \in K$.

Let $b \in D$ and $1<i \leqslant N$, and define $K_{b}^{i}=\left\{\left(a_{i}, \ldots, a_{N}\right) \in A^{N}=K: a_{i}=b\right\}$. The set $K_{b}^{i}$ is $w^{*}$-closed and open, hence the function $\mu \rightarrow \mu\left(K_{b}^{i}\right)$ is $w^{*}$-continuous.

We claim that for each $b$ and $i$, there is a finite subset $D(b, i)$ of $D$ so that

$$
\varphi\left(\xi\left(a_{1}, \ldots, a_{N}\right)\right)\left(K_{b}^{i}\right)>1-1 / N^{2}
$$

whenever $a_{i}=b$ and $a_{j} \notin D(b, i)$ for all $j \neq i$.

Indeed, if this were false, then there are points $\bar{a}_{k}=\left(a_{1}^{k}, \ldots, a_{N}^{k}\right)$ with $\varphi\left(\xi\left(\bar{a}_{k}\right)\right)\left(K_{b}^{i}\right)<1-1 / N^{2}, a_{i}^{k}=b$ and $a_{j}^{k} \neq a_{j}^{l}$ for all $k \neq l$ and $j \neq i$. But then $a_{j}^{k} \rightarrow_{k \rightarrow \infty} p$ for all $j \neq i$, and the definition of $\xi\left(\bar{a}_{k}\right)$ implies that $\xi\left(\bar{a}_{k}\right) \rightarrow$ $\delta_{(p, \ldots, p, b, p, \ldots, p)}$, where $b$ appears on the $i$ th coordinate. Thus

$$
\varphi\left(\xi\left(\bar{a}_{k}\right)\right) \stackrel{w^{*}}{\rightarrow} \varphi\left(\delta_{(p, \ldots, b, \ldots, p)}\right)=\delta_{(p, \ldots, b, \ldots, p)}
$$


and

$$
1-1 / N^{2} \geqslant \varphi\left(\xi\left(\bar{a}_{k}\right)\right)\left(K_{b}^{i}\right) \rightarrow \delta_{(p, \ldots, b, \ldots, p)}\left(K_{b}^{i}\right)=1,
$$

a contradiction.

Let $\psi(b)=D \backslash \cup_{i=1}^{N} D(b, i)$. For each $b \in D, \psi(b)$ is a subset of $D$ with a finite complement, and we shall use the following combinatorial lemma (for a proof, see e.g. [5] where the lemma is also used in a similar way):

LEMMA 5. Let $D$ be an uncountable set, and $N$ an integer. Assume that for each $b \in D, \psi(b)$ is a subset of $D$ with a finite complement. Then there are $\left\{b_{i}\right\}_{i=1, \ldots, N}$ in $D$ with $b_{i} \in \psi\left(b_{j}\right)$ for all $i \neq j$.

Using Lemma 5 , we can find $\bar{b}=\left(b_{1}, \ldots, b_{N}\right)$ so that $\varphi(\xi(\bar{b}))\left(K_{b_{b}}^{i}\right)>1-1 / N^{2}$ for all $i=1, \ldots, N$. Since $\|\varphi(\xi(\bar{b}))\| \leqslant 1$, we get that $\varphi(\xi(\bar{b}))\left(K \backslash K_{b_{i}}^{i}\right)<1 / N^{2}$ for all $i \leqslant N$, hence

$$
\varphi(\xi(\bar{b}))\left(\bigcap_{i=1}^{N} K_{b_{i}}^{i}\right)>1-N \cdot 1 / N^{2}=1-1 / N .
$$

But $\cap_{i=1}^{N} k_{b_{i}}=\{\bar{b}\}$, thus $\varphi(\xi(\bar{b}))=\alpha \delta_{\bar{b}}+\mu$ where $\alpha>1-1 / N$ and $\|\mu\|<1 / N$, and consequently

$$
\begin{aligned}
\|\varphi(\xi(\bar{b}))-\xi(\bar{b})\| & >\alpha+\|\xi(\bar{b})\|-\|\mu\| \\
& \geqslant \frac{N+1}{N-1}+1-2 / N>2
\end{aligned}
$$

4. Remarks and open problems. (1) Proposition 1 raises natural questions on the possible "regularity" with respect to the norm topology, of a $w^{*}$-continuous retract on the unit ball of the dual of a separable Banach space. To the best of our knowledge this subject has not been treated in the literature.

(2) The problem treated here makes sense for other classical Banach spaces. For example, it is proved in [2] that for every $1 \leqslant p<\infty, p \neq 2$ there is a constant $\varepsilon(p)$ so that if $L_{p}(\mu)$ and $L_{p}(\nu)$ are separable and isomorphic, with an isomorphism $T$ satisfying $\|T\|\left\|T^{-1}\right\| \leqslant 1+\varepsilon$ with $\varepsilon<\varepsilon(p)$, then they are in fact isometric (see also [4]). We are thus led to the following problem: Is there a function $g(\varepsilon)$, with $g(\varepsilon) \rightarrow_{e \rightarrow 0} 0$ so that if $\mu, \nu, p$ and $T$ are as above, there is an isometry of $L_{p}(\mu)$ onto $L_{p}(\nu)$ with $\|T-W\| \leqslant g(\varepsilon)$ ? Does the same hold for INTO-isomorphisms?

(3) As Theorem 5 shows, one cannot generalize Theorem 2 to the nonmetrizable case. A weaker generalization would be that if there is an isomorphism $T$ of $C(K)$ into $C(S)$ with $\|T\|\left\|T^{*}\right\|<1+\varepsilon$ with $\varepsilon$ small enough, then $C(K)$ is isometric to a subspace of $C(S)$ (without any estimate on the distance between the isometry and $T)$. We conjecture that this is also false in general.

ADDED IN PROOF. Two of the problems indicated in $\$ 4$ have been dealt with since the submission of this paper.

(1) In Simultaneously continuous retractions on the unit ball of a Banach space, we study in detail the possible norm-regularity of a $\omega^{*}$-continuous retract from $X^{*}$ onto its unit ball.

(2) The analogue problem for $L_{p}(\mu)$ spaces, is solved positively by D. E. Alspach in Small into-isomorphisms between $L^{p}$ spaces. 


\section{REFERENCES}

1. D. Amir, On isomorphism of continuous function spaces, Israel J. Math. 3 (1965), 205-210.

2. Y. Benyamini, Near isometries in the class of $L^{1}$-preduals, Israel J. Math. 20 (1975), 275-281.

3. M. Cambern, On isomorphism with small bounds, Proc. Amer. Math. Soc. 18 (1967), $1062-1066$.

4. __ On $L^{1}$ isomorphisms, Proc. Amer. Math. Soc. 78 (1980), 227-228.

5. H. H. Corson and J. Lindenstrauss, On simultaneous extension of continuous functions, Bull. Amer. Math. Soc. 71 (1965), 542-545.

6. J. Lindenstrauss and L. Tzafriri, Classical Banach spaces, Lecture Notes in Math., vol. 338, Springer-Verlag, Berlin and New York, 1973.

Department of Mathematics, Technon-Israel Institute of Technology, Haifa, Israel 